\title{
Intoxicação experimental pelos frutos de Xanthium cavanillesii (Asteraceae) em bovinos ${ }^{1}$
}

\author{
Edson M. Colodel ${ }^{2}$, David Driemeier ${ }^{3}$ e Célso Pilati ${ }^{4}$
}

\begin{abstract}
Colodel E.M., Driemeier D. \& Pilati C. 2000. [Experimental poisoning by the burs of Xanthium cavanillesii (Asteraceae) in cattle.] Intoxicação experimental pelos frutos de Xanthium cavanillesii em bovinos. Pesquisa Veterinária Brasileira 20(1):31-38. Depto Clínica Médica Veterinária, Faculdade de Agronomia e Medicina Veterinária, UFMT, Av. Fernando Correa da Costa, Cuiabá, MT 78010-900, Brazil.

The ground burs of Xanthium cavanillesii Schouw were force fed to 11 calves in single or repeated doses. Quantities of $5 \mathrm{~g} / \mathrm{kg}$ and above were lethal and 4 animals died. A single dose of $3 \mathrm{~g} / \mathrm{kg}$ caused moderate clinical signs in 1 calf. Weekly doses of $3 \mathrm{~g} / \mathrm{kg}$ and $5 \mathrm{~g} / \mathrm{kg}$ fed to 2 calves during 4 weeks and 2 weeks, respectively, did not cause poisoning. Hypoglicemia and increased levels of aspartate aminotransferase (AST) were detected only in the animals that showed clinical signs. These were observed between 7 hours and 12 hours after the beginning of the administration of the burs and included apathy, excessive salivation, generalized muscle tremors, trismus, diffuse sweating and decreased rate and intensity of ruminal movements. Locomotor disturbances consisted of stiff and incoordinated gait, unwillingness to move, and recumbency. In the terminal stages, there were convulsive seizures, paddling movements, muscle spasms, apnea and death. One animal recovered from the disease. In this case, the clinical picture was similar to the one of the animals that died, although less intense. The first signs were observed 18 hours after the beginning of the administration and lasted 72 hours. Liver biopsies from that animal revealed coagulative hepatocellular necrosis associated with congestion and hemorrhages. The degenerative and necrotic hepatocellular changes were less intense with longer evolution of the clinical picture. In the calf that died, microscopic lesions were observed in liver biopsies 12 hours after the beginning of administration. Serum analysis releaved decreased glucose levels and increased aspartate aminotransferase seric activity. Main necropsy findings included accentuation of the lobular pattern of the liver and marked edema of the gall bladder wall and in adjacent tissues. The contents of omasum and rectum were dried up. There was also ascites, and disseminated petechiae and ecchymoses on serous membranes. The main histopathological changes consisted of marked coagulative hepatocellular necrosis which varied from centrilobular to massive, and was associated with congestion and hemorrhage. In the remaining of the hepatic lobule there was either swelling or vacuolation of the hepatocytes.
\end{abstract}

INDEX TERMS: Plant poisoning, Xanthium cavanillesii, cattle, hepatocellular necrosis.

\footnotetext{
${ }^{1}$ Aceito para publicação em 22 de setembro de 1999.

Parte da tese de mestrado do primeiro autor, Faculdade de Veterinária, Universidade Federal do Rio Grande do Sul (UFRGS), Porto Alegre, RS.

${ }^{2}$ Depto Clínica Médica Veterinária, Faculdade de Agronomia e Medicina Veterinária, Universidade Federal de Mato Grosso (UFMT), Av. Fernando Correia da Costa, Coxipó, Cuiabá, MT 78010-900.

${ }^{3}$ Depto Patologia Clínica Veterinária, Fac. Vet., UFRGS, Caixa Postal 15094, Porto Alegre, RS 91540-000; bolsista do CNPq (301076/93-6).

${ }^{4}$ Centro de Ciências Agroveterinárias, Universidade do Estado de Santa Catarina (CAV/UDESC), Av. L. Camões 2090, Lages, SC 88520000.
}

RESUMO.- Os frutos moídos de Xanthium cavanillesii Schouw, foram administrados por via oral, em doses única ou repetidas, com intervalo semanal, a onze bovinos. Desses, quatro morreram. Doses únicas a partir de $5 \mathrm{~g} / \mathrm{kg}$ foram letais para bovinos. Dose de $3 \mathrm{~g} / \mathrm{kg}$ produziu sinais clínicos e recuperação em um bovino. Repetições de 4 doses de $3 \mathrm{~g} / \mathrm{kg}$ para um bovino e 2 doses de $5 \mathrm{~g} / \mathrm{kg}$ para outro bovino não foram tóxicas. Foram constatadas hipoglicemia e elevação dos níveis séricos de aspartato aminotransferase (AST) nos bovinos que apresentaram sinais clínicos da intoxicação. Os primeiros sinais clínicos nos animais que morreram foram observados 
entre 6 e 12 horas após a administração dos frutos. A evolução do quadro clínico variou entre $5 \mathrm{~h} 30 \mathrm{~min}$ e 8 horas. O quadro clínico foi semelhante nestes animais sendo que os principais sinais clínicos foram anorexia, apatia, salivação profusa e tremores musculares. Ocorreram também hipomotilidade e atonia ruminal, cólicas abdominais, gemidos freqüentes, ranger de dentes, sudorese generalizada e endoftalmia. As alterações de locomoção observadas foram incoordenação motora, instabilidade do trem posterior, decúbito permanente com movimentos de pedalagem, espasmos musculares e opistótono. As alterações respiratórias foram aumento da frequiência respiratória, respiração laboriosa com ruídos e momentos de apnéia. Finalmente ocorria perda do reflexo palpebral, ausência de reflexo pupilar e morte. No bovino que se recuperou, os primeiros sinais clínicos foram observados 18 horas após a administração e evoluíram num período de aproximadamente 72 horas. Neste bovino, através de biópsias hepáticas, observou-se necrose hepática coagulativa centrolobular associada a congestão e hemorragias. Necrose hepática coagulativa massiva foi observado por biópsias hepáticas em um bovino que morreu, a partir de 12 horas após a administração dos frutos, associada com alterações nos níveis séricos de glicose e AST. As principais lesões encontradas na necropsia foram no fígado e consistiam de aumento do padrão lobular na superfície capsular e de corte, distensão da vesícula biliar e edema moderado da parede da vesícula biliar. A principal alteração microscópica era caracterizada por necrose coagulativa centrolobular ou massiva associada a congestão e hemorragia e alterações degenerativas nos hepatócitos circunjacentes.

TERMOS DE INDEXAÇÃO: Plantas tóxicas, Xanthium cavanillesii, bovinos, necrose hepatocelular.

\section{INTRODUÇÃO}

As intoxicações por plantas do gênero Xanthium ocorrem em animais domésticos pela ingestão da brotação, em condições de carência de pastagens (Méndez et al. 1998), ou pela ingestão de frutos que acidentalmente contaminam a alimentação de animais (Witte et al. 1990, Driemeier et al. 1999). O quadro clínico-patológico de intoxicação por Xanthium spp. caracteriza-se por necrose hepática, causando sinais clínicos e morte associadas à insuficiência hepática aguda. Carboxiatractilosídeo, um glicosídeo hepatotóxico, é isolado dos frutos e da brotação da planta (Stuart et al. 1981).

A utilização de subprodutos agro-industriais é uma prática freqüente na pecuária brasileira. $O$ gênero Xanthium é um invasor que infesta lavouras de culturas anuais, sendo difícil evitar a contaminação da colheita pelos frutos dessa planta. Quando subprodutos contaminados com os frutos de Xanthium sp. são utilizados como alimentos para animais, podem trazer prejuízos econômicos.

No Estado do Rio Grande do Sul há relatos de intoxicação por resíduos de lavoura de soja contaminados com frutos de Xanthium cavanillesii, causando mortalidade de bovinos em pelo menos três municípios: Casca, Cachoeira (Driemeier et al. 1999) e Pinhal Grande (Loretti et al. 1999), com aproxima- damente $8 \%$ de mortalidade nos rebanhos afetados. Dados sobre a intoxicação pelos frutos de Xanthium spp. são escassos em bovinos. $\mathrm{O}$ objetivo deste trabalho foi determinar a toxidez dos frutos de $X$. cavanillesii, estabelecer a dose tóxica, determinar o quadro clínico-patológico e as alterações bioquímicas associadas com a intoxicação em bovinos. Foi feito estudo através de biópsias hepáticas sobre o desenvolvimento das lesões hepáticas microscópicas. Estes dados visam facilitar o diagnóstico da intoxicação natural e a tomada de medidas profiláticas que minimizem as perdas econômicas dos rebanhos afetados.

\section{MATERIAL E MÉTODOS}

Nesse trabalho experimental foram utilizados 11 bovinos, 5 machos e 6 fêmeas, sem raça definida, com idade entre 5 e 24 meses. Todos os bovinos eram pesados antes de iniciar o experimento (Quadro 1). Durante os experimentos os animais ficavam presos em baias

Quadro 1. Delineamento da intoxicação experimental pelos frutos de Xanthium cavanillesii em bovinos

\begin{tabular}{cccccc}
\hline Bovino & $\begin{array}{c}\text { Idade } \\
\text { (meses) }\end{array}$ & $\begin{array}{c}\text { Peso } \\
(\mathrm{kg})\end{array}$ & Sexo & $\begin{array}{c}\text { Administração } \\
\mathrm{g} / \mathrm{kg}\end{array}$ & $\begin{array}{c}\text { Número de } \\
\text { administrações }\end{array}$ \\
\hline 1 & 07 & 150 & Macho & 03,0 & $4^{\mathrm{a}}$ \\
2 & 24 & 275 & Fêmea & 05,0 & $2^{\mathrm{a}}$ \\
3 & 12 & 109 & Fêmea & 10,0 & $\mathrm{DU}^{\mathrm{b}}$ \\
4 & 08 & 160 & Fêmea & 03,0 & DU \\
5 & 14 & 220 & Fêmea & 01,5 & DU \\
6 & 12 & 150 & Macho & 10,0 & DU \\
7 & 14 & 180 & Fêmea & 15,0 & DU \\
8 & 12 & 215 & Macho & 01,5 & DU \\
9 & 12 & 225 & Macho & 05,0 & DU \\
10 & 12 & 250 & Fêmea & 05,0 & DU \\
11 & 05 & 57 & Macho & 03,0 & DU
\end{tabular}

a Com intervalos semanais.

b Dose única.

individuais de alvenaria, com piso de cimento. Eram soltos diariamente para observação em piquete de aproximadamente 2 hectares com pastagem de azevém, trevo e pasto nativo. Durante o período de experimentação, os bovinos eram alimentados com silagem de sorgo, resíduo de cervejaria e água ad libitum. Os frutos de Xanthium cavanillesii Schouw utilizados no experimento eram procedentes de resíduos de lavoura de soja que causaram mortalidade de bovinos em estabelecimentos localizados nos municípios de Casca e Cachoeira do Sul (Driemeier et al. 1999). Os frutos de X. cavanillesii foram separados manualmente do resíduo e guardados em temperatura ambiente. Os frutos de $X$. cavanillesii, eram triturados em moinho martelo, malha 20. Antes da administração o material triturado era misturado com água, até tornar o triturado de consistência pastosa, e fornecido aos bovinos para ingestão voluntária ou através de ingestão forçada por via oral.

Exames clínicos foram realizados antes da administração e duas vezes por dia após a administração dos frutos, com intervalos de aproximadamente 8 horas. $O$ acompanhamento dos animais experimentais era feito com maior freqüência quando eram observadas alterações de comportamento. Nos exames clínicos eram avaliados os seguintes parâmetros: apetite, sede, aspecto das fezes e da urina, movimentos ruminais, frequiências cardíaca e respiratória, temperatura retal, coloração 
Quadro 2. Biópsias hepáticas na intoxicação experimental pelos frutos de Xanthium cavanillesii em bovinos

\begin{tabular}{|c|c|c|}
\hline Bovino & Coleta & $\begin{array}{c}\text { Período de } \\
\text { coleta }^{\mathrm{a}} \text { (horas) }\end{array}$ \\
\hline \multirow[t]{6}{*}{4} & $1^{o}$ & 51 \\
\hline & $2^{\circ}$ & 69 \\
\hline & $3^{\circ}$ & 92 \\
\hline & $4^{\circ}$ & 116 \\
\hline & $5^{\circ}$ & 260 \\
\hline & $6^{\circ}$ & b \\
\hline \multirow[t]{3}{*}{6} & $1^{\mathrm{o}}$ & $0^{c}$ \\
\hline & $2^{\circ}$ & 51 \\
\hline & $3^{\circ}$ & 95 \\
\hline \multirow[t]{5}{*}{9} & $1^{\mathrm{o}}$ & 0 \\
\hline & $2^{\circ}$ & 4 \\
\hline & $3^{\circ}$ & 8 \\
\hline & $4^{\circ}$ & 12 \\
\hline & $5^{\circ}$ & 16 \\
\hline \multirow[t]{3}{*}{11} & $1^{\mathrm{o}}$ & 0 \\
\hline & $2^{\circ}$ & 24 \\
\hline & $3^{\circ}$ & 48 \\
\hline
\end{tabular}

aPosterior à administração de $X$. cavanillesii.

besta coleta foi realizada aproximadamente 1 ano após a administração.

${ }^{\mathrm{c} A}$ hora 0 (zero), nesta coluna, refere-se à realização de biópsia antes da administração de frutos de $X$. cavanillesii.

das mucosas oral e conjuntival, alterações cárdio-respiratórias, modificações de comportamento, postura e movimentação.

Foram feitas biópsias hepáticas pelo método de punção transtorácica usando-se agulha de Menghini (Braga et al. 1985). Os bovinos, número de biópsia por animal e o tempo de realização de biópsia relacionado à administração de frutos de $X$. cavanillesii estão resumidos no Quadro 2. O material de biópsia foi coletado em solução de formalina $10 \%$ tamponada e processada para exame histológico. Nos Bovinos 6, 7 e 9 foram feitas coletas de sangue para mensuração da atividade sérica das enzimas aspartartato aminotransferase (AST) e fosfatase alcalina (SAP). Foi mensurado o nível sérico de glicose nos Bovinos 1, 6, 7 e 9 .

Nos Bovinos 1 e 2 que foram sacrificados e nos Bovinos 3, 7, 9 e 10 que morreram em conseqüência da intoxicação, foram feitas necropsias. Fragmentos de fígado, rim, adrenal, baço, pâncreas, linfonodos mediastínicos, bexiga, tubo digestivo, pulmão e coração foram coletados e fixados em formalina 10\%. Amostras dos fragmentos foram processadas rotineiramente e corados pela técnica de hematoxilina e eosina (HE). $O$ encéfalo foi coletado inteiro e fixado em formalina tamponada $10 \%$. Fragmentos de quatro regiões anatômicas foram sistematicamente processados para exame histológico. Estas regiões incluíram o córtex cerebral sobre o diencéfalo, tálamo, corpos quadrigêmeos e cerebelo com pedúnculos cerebelares e bulbo.

\section{RESULTADOS}

\section{Sinais clínicos}

A evolução do quadro clínico observado na intoxicação experimental pelos frutos de $X$. cavanillesii está resumido no Quadro 3.

Sinais clínicos de intoxicação foram observados em 5 bovinos (Bov. 3, 4, 7, 9 e 10), desses apenas o Bovino 4 não
Quadro 3. Intoxicação experimental pelos frutos de Xanthium cavanillesii em bovinos

\begin{tabular}{ccccc}
\hline Bovino & $\begin{array}{c}\text { Dose } \\
(\mathrm{g} / \mathrm{kg})\end{array}$ & $\begin{array}{c}\text { Início dos } \\
\text { sinais clínicos }\end{array}$ & $\begin{array}{c}\text { Evolução clínica } \\
\text { (horas) }\end{array}$ & Desfecho \\
\hline 1 & $3,0 \times 4$ & Sem sinais & - & Não adoeceu \\
2 & $5,0 \times 2$ & Sem sinais & - & Não adoeceu \\
Morreu \\
3 & 10,0 & $7 \mathrm{~h}^{\mathrm{a}}$ & $8 \mathrm{~h}$ & $\begin{array}{c}\text { Recuperou-se } \\
4\end{array}$ \\
3,0 & $18 \mathrm{~h}$ & $72 \mathrm{~h}$ & Não adoeceu \\
5 & 1,5 & Sem sinais & - & Não adoeceu \\
6 & 10,0 & Sem sinais & - & Morreu \\
7 & 15,0 & $7 \mathrm{~h}$ & $5 \mathrm{~h} 30 \mathrm{~min}$ & Não adoeceu \\
8 & 1,5 & Sem sinais & - & Morreu \\
9 & 5,0 & $12 \mathrm{~h}$ & $7 \mathrm{~h}$ & Morreu \\
10 & 5,0 & $7 \mathrm{~h}$ & $6 \mathrm{~h}$ & Não adoeceu \\
11 & 3,0 & Sem sinais & - &
\end{tabular}

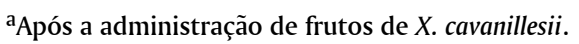

morreu. A menor dose que causou morte foi $5 \mathrm{~g} / \mathrm{kg}$ (Bov. 9 e 10). A dose de $1,5 \mathrm{~g} / \mathrm{kg}$ não causou sinais clínicos nos animais testados (Bov. 5 e 8); a de 3g/kg causou sinais clínicos e recuperação em um bovino (Bov. 4). $O$ Bovino 1 que recebeu 4 doses de $3 \mathrm{~g} / \mathrm{kg}$ e o Bovino 2 que recebeu 2 doses de $5 \mathrm{~g} / \mathrm{kg}$, com intervalo de 7 dias cada, não apresentaram sinais clínicos. O Bovino 6 recebeu dosagem de $10 \mathrm{~g} / \mathrm{kg}$ e não desenvolveu sinais clínicos

Os primeiros sinais clínicos observados nos casos fatais variaram de 6 a 12 horas após a administração dos frutos de $X$. cavanillesii. A evolução clínica oscilou de 5 horas e meia a 8 horas. No bovino que não morreu, primeiros sinais foram observados 18 horas após a administração e a evolução do início dos sinais até os últimos sinais clínicos foi de aproximadamente 72 horas.

O quadro clínico foi semelhante nestes animais e consistiu de alterações digestivas, respiratórias e neuromusculares. Inicialmente foram constatadas anorexia e apatia. Os animais movimentavam-se lentamente, mantinham a cabeça baixa, as orelhas caídas e mostravam-se alheios ao ambiente. Salivação profusa foi observada já no início dos sinais clíni$\cos$ nos Bovinos 7 e 9. Tremores musculares, inicialmente eram observados na forma de tremores leves principalmente nos músculos da face e do pescoço. Posteriormente tornavam-se generalizados, sendo mais evidentes nos músculos dos membros, persistindo até próximo da morte. Mioclonias ocorreram nos músculos da face e dos membros. Havia diminuição progressiva dos movimentos ruminais e posterior atonia ruminal. Os bovinos apresentavam sinais de dor abdominal caracterizados pela face hipocrática, resistência à movimentação, decúbitos freqüentes, olhar freqüente ao abdômen, bater a cabeça contra o costado, batidas constantes dos cascos principalmente do trem posterior contra o piso (Bov. $3 \mathrm{e}$ 7). Foram observados gemidos e algumas vezes mugidos, principalmente durante a micção e defecação. Os gemidos eram frequientes ocorrendo até próximo à morte dos animais. Também foram notados ranger de dentes, sudorese generalizada, mais evidente na cabeça e região cervical, e endoftalmia leve a moderada (Bov. 7, 9 e 10). As alterações de locomoção observadas foram caracterizadas pela dificuldade em cami- 


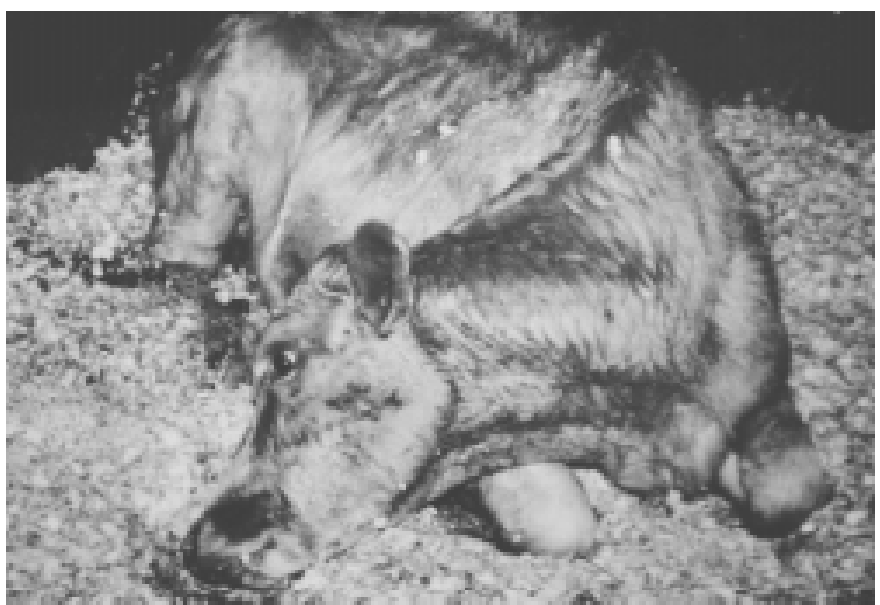

Fig. 1. Decúbito esternal, com a cabeça voltada para trás e apoiando o queixo no chão, boca levemente aberta, pêlos da região cervical úmidos devido à sudorese, na intoxicação experimental pelos frutos de Xanthium cavanillesii em bovinos (Bov. 9).

nhar, arrastar as pinças e instabilidade do trem posterior. Os bovinos deitavam-se lentamente e com dificuldade. Para levantarem-se, inicialmente erguiam os membros posteriores, ficando algum tempo com os anteriores flexionados mostrando sinais de fraqueza. Posteriormente assumiam decúbito esternal permanente. Voltavam freqüentemente a cabeça em direção ao abdômen e apoiavam o focinho ou o mento no piso da baia (Fig. 1). Alterações respiratórias caracterizavamse por aumento da frequiência respiratória, respiração laboriosa e ruidosa com curtos períodos de apnéia depois da expiração. Posteriormente assumiam decúbito lateral. Não conseguiam manter o decúbito esternal mesmo quando ajudados. Quadros convulsivos ocorreram neste período e foram caracterizados por movimentos de pedalagem rápidos $\mathrm{e}$ vigorosos, alternando entre pedalagem dos membros anteriores e posteriores ou apenas dos anteriores. Entre esses movimentos de pedalagem eram observados espasmos tetânicos acentuados nos membros anteriores e posteriores. Os espasmos musculares eram mais fortes nos posteriores. A intensidade e frequiência de espasmos diminuíam próximos à morte. $\mathrm{O}$ Bovino 7 apresentou hipotermia aproximadamente 1 hora antes da morte. Nessa fase foi notado que os bovinos voltavam progressivamente a cabeça para trás e apresentavam fluxo pequeno e contínuo de espuma branca pela boca. Finalmente observavam-se períodos de apnéia acompanhados de inspiração profunda com gemidos, ranger de dentes, e morte.

O Bovino 4, que se recuperou, apresentou o quadro clínico semelhante ao dos animais que morreram, porém, os sinais clínicos foram menos intensos. Os primeiros sinais foram observados 18 horas após a administração e evoluíram num período de aproximadamente 72 horas. Inicialmente ocorreu diminuição do apetite evoluindo para anorexia total e depressão. Apresentava apatia e tremores musculares. Deitava e levantava-se com frequiência, apresentava sialorréia leve e contínua, sudorese leve e pêlos arrepiados. Após 25 horas

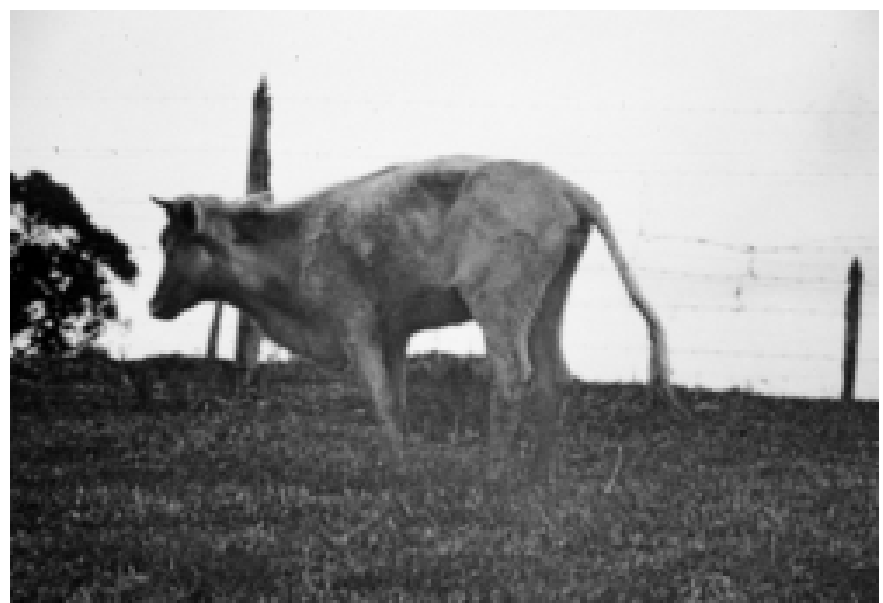

Fig. 2. Sinais de tenesmo (cauda levantada, membros aproximados) na intoxicação experimental pelos frutos de $X$. cavanillesii em bovinos (Bov. 4).

da administração, os tremores musculares eram generalizados e bem evidentes. O bovino ficava por longos períodos em decúbito esternal, fazia várias tentativas para se levantar, sem êxito, antes de conseguir permanecer em estação. Quando em estação, movimentava-se lentamente. Solto no piquete apresentava marcha normal, porém cansava com facilidade. Após movimentação foi observada intensificação dos tremores musculares e salivação. Após 40 horas de evolução clínica, a frequiência respiratória e os movimentos ruminais diminuíram e os tremores musculares eram menos evidentes. Apresentava andar rígido, lento, cambaleante e arrastava as pinças. Com 60 horas este animal tinha dificuldades em acompanhar outros bovinos do rebanho, caminhando lentamente com a cauda levantada (Fig. 2). As fezes eram escassas, com consistência firme, envoltas em muco e com estrias de sangue. Os sinais clínicos regrediram gradativamente, cessando os tremores, a salivação, a anorexia e a depressão. Com 90 horas após a administração da planta este animal não aparentava mais alterações clínicas.

\section{Achados de análises clínicas}

Os resultados da mensuração dos níveis de glicose sérica e dos marcadores enzimáticos de lesão hepática, aspartato aminotransferase (AST) e fosfatase alcalina (SAP), são apresentados no Quadro 4.

Foram feitas dosagens dos níveis de glicose sérica nos Bovinos 1, 6, 7 e 9. Nos Bovinos 1 e 6 não ocorreram alterações nos níveis de glicose sérica fora dos padrões de normalidade, após as administrações dos frutos de $X$. cavanillesii. No Bovino 7 a primeira aferição, com níveis de glicose abaixo dos valores normais, foi obtida aproximadamente 12 horas após a administração de frutos de $X$. cavanillesii. Este bovino demonstrava sinais clínicos há aproximadamente 5 horas. $O$ resultado da dosagem anterior deste animal, realizada 3 horas após o início dos sinais clínicos, estava no limiar mínimo da normalidade. No Bovino 9 foram constatados níveis de glicose abaixo dos parâmetros 
Quadro 4. Dosagens séricas de glicose e enzimas aspartato aminotransferase (AST) e fosfatase alcalina (SAP), na intoxicação experimental pelos frutos de Xanthium cavanillesii em bovinos

\begin{tabular}{|c|c|c|c|c|c|}
\hline Bovino & Coleta & Tempo $^{a}$ & $\begin{array}{c}\text { Glicose } \\
\mathrm{mg} / \mathrm{dl}\end{array}$ & AST/UI/L & SAP/UI/I \\
\hline \multirow[t]{5}{*}{1} & $1^{b}$ & $0^{d}$ & 97 & - & - \\
\hline & $2^{b}$ & 6 & 76 & - & - \\
\hline & $3^{b}$ & 22 & 82 & - & - \\
\hline & $4^{c}$ & 0 & 72 & - & - \\
\hline & $5^{c}$ & 8 & 71 & - & - \\
\hline \multirow[t]{2}{*}{6} & 1 & 0 & 67 & 33 & 154 \\
\hline & 2 & 15 & 68 & 67 & 220 \\
\hline \multirow[t]{5}{*}{7} & 1 & 0 & 54 & 246 & 104 \\
\hline & 2 & 8 & 45 & 1297 & 88 \\
\hline & 3 & 12 & 32 & 2047 & 84 \\
\hline & 4 & 13 & 25 & 1311 & 56 \\
\hline & 5 & 15 & 30 & 1235 & 440 \\
\hline \multirow[t]{4}{*}{9} & 1 & 0 & 76 & 96 & 84 \\
\hline & 2 & 13 & 33 & 8860 & 418 \\
\hline & 3 & 15 & 30 & 8370 & 362 \\
\hline & 4 & 19 & 29 & 6475 & 301 \\
\hline
\end{tabular}

aTempo em horas decorrido após a administração de frutos de X. cavanillesii. bPrimeira administração.

'Segunda administração (intervalo semanal entre administrações).

"Os valores "0" constantes nesta coluna são relacionados à coleta de soro sangǘneo antes da administração de $X$. cavanillesii.

normais 1 hora após o início dos sinais clínicos e permaneceram abaixo da normalidade até a morte.

Alterações nos níveis de AST ocorreram apenas nos animais que apresentaram sinais clínicos. Nos Bovinos 7 e 9 foram constatadas alterações 45 minutos e 1 hora, respectivamente, após o início dos sinais clínicos, permanecendo elevadas até o óbito dos animais. A atividade sérica da enzima

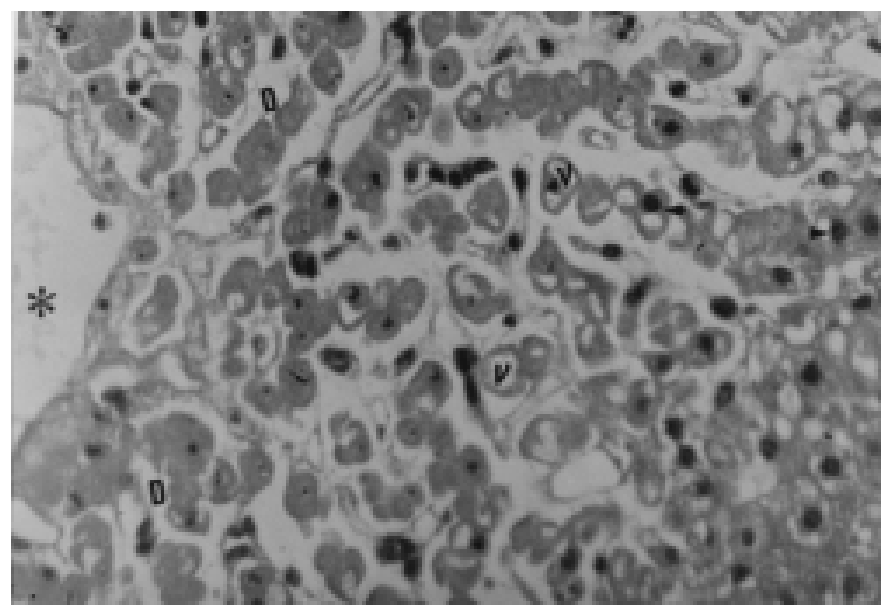

Fig. 3. Biópsia hepática na intoxicação experimental pelos frutos de X. cavanillesii em bovinos (Bov. 9): Necrose coagulativa massiva; dilatação do espaço de Disse (D), hepatócitos diminuídos em volume, com citoplasma eosinofílico, vacúolos citoplasmáticos de aspecto vesicular (V); núcleos em picnose (ponta de seta) e cariorrexia (seta), veia centrolobular ("). HE, obj. 40.
SAP não mostrou alterações além dos valores normais nos três bovinos que foram submetidos a avaliação deste parâmetro.

\section{Biópsias hepáticas}

No Bovino 9 foram feitas cinco biópsias. Macroscopicamente, constatou-se acentuação do padrão lobular a partir da $4^{a}$ coleta. Microscopicamente as alterações foram observadas 12 horas após a administração sendo caracterizadas por hepatócitos centrolobulares separados entre si, diminuídos de tamanho e com o núcleo picnótico ou, com menor frequiência, cariorrético. O citoplasma dos hepatócitos necróticos mostrava-se fortemente eosinofílico, homogêneo ou granular e ocasionalmente vacuolizado. Na $5^{\mathrm{a}}$ coleta, 16 horas após a administração, foi constatada necrose hepática massiva caracterizada por aumento de eosinofilia do citoplasma hepatocelular e grande quantidade de núcleos cariorréticos (Fig. 3). No Bovino 4, nas cinco primeiras biópsias foram constatadas alterações macroscópicas nos fragmentos coletados. Essas consistiam em acentuação do padrão lobular caracterizada por áreas vermelho-escuras intercaladas com áreas alaranjadas. Microscopicamente essas alterações correspondiam à necrose hepática coagulativa centrolobular associada à congestão e hemorragias. Havia acúmulo de células inflamatórias; principalmente mononucleares nos sinusóides, e áreas de necrose. $\mathrm{O}$ citoplasma das células necróticas aparecia vacuolizado. Na região mediozonal foram constatadas alterações degenerativas caracterizadas por tumefação e vacuolização do citoplasma e do núcleo de hepatócitos. As principais diferenças entre essas sequiências de biópsias foram na intensidade de hemorragia e congestão; essas eram moderadas a acentuadas na primeira biópsia e discretas na quinta coleta. Esse achado foi associado a aumento gradativo de pigmento acastanhado e refringente no

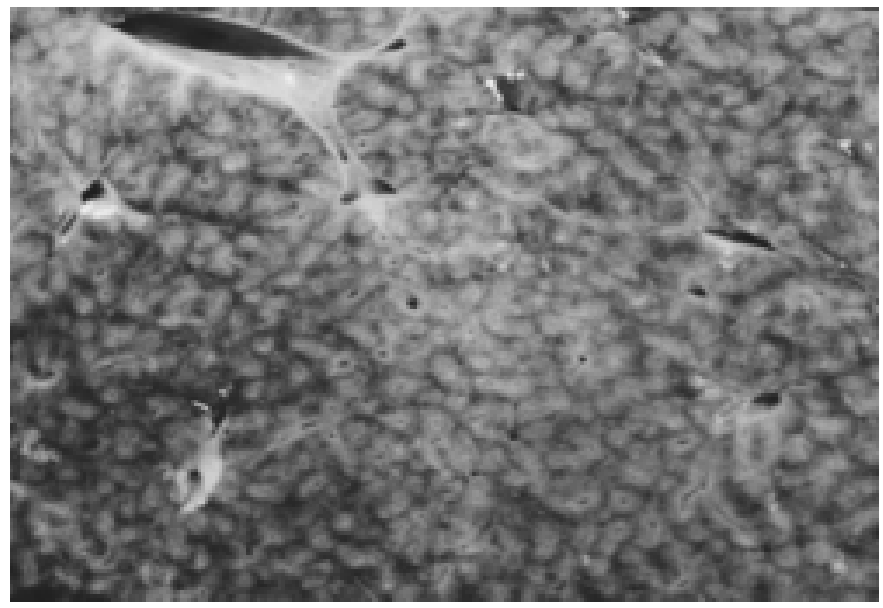

Fig. 4. Acentuação do padrão lobular caracterizada por áreas alaranjadas intercaladas com áreas avermelhadas na intoxicação experimental pelos frutos de X. cavanillesii em bovinos (Bov. 9). 
citoplasma de macrófagos na área de necrose. Gradativamente foi constatado desaparecimento da necrose centrolobular; na quinta coleta atingia duas a três fileiras de hepatócitos adjacentes à veia centrolobular. $\mathrm{Na}^{6}{ }^{\underline{a}}$ biópsia, realizada aproximadamente um ano após a administração de $X$. cavanillesii, não foram observadas alterações microscópicas.

\section{Achados de necropsia}

As principais lesões encontradas na necropsia estavam localizadas no fígado dos Bovinos 3, 7, 9 e 10 e consistiam em acentuação do padrão lobular nas superfícies capsular e de corte. Na superfície de corte foram observadas áreas alaranjadas e pálidas intercaladas com áreas vermelho-escuras conferindo à superfície um aspecto de noz-moscada (Fig. 4). Nos Bovinos 3 e 7 esta alteração era mais evidente no lobo caudado e homogênea nos demais lobos. Na superfície subcapsular e de corte eram encontradas hemorragias petequiais de distribuição aleatória. Nesses bovinos, a vesícula biliar estava distendida com conteúdo de baixa viscosidade. Edema moderado da parede da vesícula biliar, do colédoco, da superfície serosa do duodeno, adjacências do esfíncter de Oddi e pâncreas foram achados frequientes. Na cavidade abdominal dos Bovinos 3 e 7 havia aproximadamente $200 \mathrm{ml}$ de líquido citrino coagulável. No Bovino 9 um coágulo sanguííneo estava localizado sobre as perfurações feitas pela agulha durante a biópsia hepática e havia aproximadamente 2 litros de sangue, não coagulado, no interior da cavidade abdominal. Nos Bovinos 3 e 10, o conteúdo do omaso estava moderadamente ressecado e a mucosa do abomaso estava difusa e intensamente avermelhada. A serosa do jejuno e íleo estava difusamente avermelhada e a mucosa adjacente apresentava petéquias e sufusões de distribuição multifocal. No reto dos Bovinos 3 e 9 as fezes eram mais consistentes, revestidas de muco e com estrias de sangue. No rim dos Bovinos 3 e 9 a cortical estava moderada e difusamente pálida. Esplenomegalia leve foi observada no Bovino 9. No coração petéquias e sufusões murais e intramiocárdicas multifocais a coalescentes foram encontradas com freqüência variando de intensidade; eram acentuadas no Bovino 10, moderadas no Bovino $3 \mathrm{e}$ leves no Bovino 7 e 9 . Congestão e edema pulmonar leve a moderado com conteúdo espumoso na traquéia foram observados nos Bovinos 3 e 9 . Os Bovinos 1 e 2 não apresentaram alterações macroscópicas significativas.

\section{Achados histopatológicos}

A principal alteração microscópica observada no fígado dos Bovinos 3, 7, 9 e 10, caracterizava-se por necrose coagulativa hepatocelular centrolobular (Bov. 3 e 7) e panlobular (massiva) (Bov. 9 e 10) associada a congestão e hemorragias. A lesão centrolobular afetava 2 terços do lóbulo hepático. A necrose panlobular afetava praticamente todo o lóbulo hepático, poupando apenas 3 ou 4 fileiras de hepatócitos aparentemente viáveis. A necrose hepatocelular era caracterizada por desorganização dos cordões de hepatócitos, individualização e diminuição do tamanho de hepatócitos, aumento da acidofilia citoplasmática e graus variáveis de vacuolização. As alterações nucleares dos hepatócitos caracterizaram-se por picnose, cariorrexia e cariólise.
Os hepatócitos da região adjacente à área de necrose apresentavam alterações degenerativas. Outras alterações no figado foram dilatação do espaço de Disse e dos sinusóides e ativação das células de Kupffer. Os sinusóides e veias centrolobulares apresentavam tumefação das células endoteliais e leve infiltrado formado por células inflamatórias mononucleares e polimorfonucleares. Nas zonas de transição entre as áreas de necrose e degeneração eram encontradas quantidades variáveis de esférulas eosinofilicas e homogêneas. Com freqüência foi observado edema no tecido conjuntivo interlobular do pâncreas, na mucosa e camada fibromuscular da vesícula biliar e na mucosa e submucosa do duodeno dos Bovinos 7, 9 e 10. Congestão difusa e moderada com áreas focais de hemorragia foram observadas na mucosa e na submucosa do abomaso, duodeno e jejuno dos Bovinos $3 \mathrm{e}$ 10. Edema pulmonar alveolar leve a moderado e difuso foi um achado frequiente. Nas adrenais do Bovino 3 foram encontradas hemorragias medulares multifocais. No rim dos Bovinos 7 e 10 foi encontrado proteinose tubular moderada. Os Bovinos 1 e 2 não apresentaram alterações histológicas significativas nos órgãos examinados

\section{DISCUSSÃO}

Neste estudo foi caracterizada a toxidez dos frutos de Xanthium cavanillesii para bovinos. De onze bovinos que receberam os frutos quatro morreram e um adoeceu e recuperou-se. O quadro clínico e os achados macroscópicos e microscópicos encontrados nos bovinos deste estudo são semelhantes aos descritos na intoxicação espontânea e experimental por plântulas de Xanthium spp. na fase com cotilédones (Witte et al. 1990, Martin et al. 1992, Méndez et al. 1998). Há também semelhanças com a intoxicação pelos frutos de $X$. cavanillesii em ovinos (Loretti 1997) e X. strumarium em suínos (Stuart et al. 1981).

A dose letal mínima de frutos de X. cavanillesii estabelecida neste experimento foi de $5 \mathrm{~g} / \mathrm{kg}$. A dose letal encontrada em dados de literatura é de aproximadamente $3 \mathrm{~g} / \mathrm{kg}$ (Kingsbury 1964, Martin et al. 1986). Neste estudo, de três bovinos que receberam $3 \mathrm{~g} / \mathrm{kg}$, dois não adoeceram e o outro recuperouse depois de apresentar sinais clínicos e alterações patológicas semelhantes aquelas observados em bovinos que morreram intoxicados por $X$. cavanillesii.

Foram realizadas mensurações bioquímicas de glicose sérica e dos níveis das enzimas aspartato aminotransferase (AST) e fosfatase alcalina (SAP). Alterações nos níveis de glicose sérica foram observadas nos Bovinos 7 e 9, achados semelhantes são descritos na intoxicação por $X$. strumarium em bovinos (Witte et al. 1990, Martin et al. 1992) e em outros animais domésticos (Stuart et al. 1981). Na necrose hepática zonal, o fígado pode tornar-se insuficiente para manter a glicemia. Esta evidência é corroborada pelo fato dos níveis de glicose estarem alterados somente após a constatação de alterações no nível da atividade sérica da AST e coincidente com alterações morfológicas hepáticas observadas através de biópsias. Não houve alteração no nível sérico de glicose nos bovinos que não apresentaram alterações clínicas e patológicas após a administração de $X$. cavanillesii. Foram cons- 
tatadas elevações dos níveis séricos de AST nos Bovinos 7 e 9. Nesse estudo a elevação acentuada da atividade sérica da AST foi atribuída a extensa necrose de hepatócitos.

Os sinais clínicos observados nos bovinos deste estudo foram compatíveis com aqueles previamente publicados sobre bovinos intoxicados natural ou experimentalmente por Xanthium spp. (Kingsbury 1964, Martin et al. 1986, Witte et al. 1990, Martin et al. 1992, Méndez et al. 1998, Driemeier et al. 1999). Neste estudo, os principais sinais observados foram digestivos, neuromusculares e respiratórios. Os principais sinais clínicos digestivos foram anorexia, hipomotilidade seguidos de atonia ruminal, tenesmo, cólicas e salivação profusa. Os sinais digestivos observados neste trabalho foram descritos em intoxicações naturais e experimentais com Xanthium spp. em bovinos (Martin et al. 1986, Méndez et al. 1998), ovinos (Loretti 1997, Méndez et al. 1998) e suínos (Stuart et al. 1981). Esses sinais clínicos ocorrem nas intoxicações de bovinos por outras plantas hepatotóxicas como Cestrum intermedium (Gava et al. 1996), Cestrum corymbosum var. hirsutum (Gava et al. 1991), Cestrum laevigatum (Döbereiner et al. 1969), Cestrum parqui (Riet-Correa et al. 1986), Sessea brasiliensis (Canella et al. 1968), Vernonia rubricaulis (Tokarnia \& Döbereiner 1982) e Vernonia mollissima (Döbereiner et al. 1976, Gava et al. 1987). Os sinais clínicos mais constantes neste estudo foram os sinais neuromusculares. Estes sinais foram caracterizados por apatia, tremores musculares, mioclonias, ranger de dentes, dificuldade locomotora, relutância em caminhar, instabilidade do trem posterior, dificuldade para se deitar e incapacidade para se levantar, sonolência, movimentos de pedalagem, convulsões e coma. A ocorrência de sinais neuromusculares em bovinos intoxicados por frutos ou brotação com cotilédones de Xanthium spp. é descrita freqüentemente em bovinos (Marsh et al. 1924, Kingsbury 1964, Martin et al. 1986, 1992, Witte et al. 1990, Driemeier et al. 1999, Loretti et al. 1999, Méndez et al. 1998), suínos e ovinos (Stuart et al. 1981, Oelkers \& Oehme 1982, Méndez et al. 1998, Loretti et al. 1999). Plantas hepatotóxicas caracteristicamente produzem sinais clínicos neuromusculares em bovinos (Canella et al. 1968, Döbereiner et al. 1969, 1976, Tokarnia \& Döbereiner 1982, Riet-Correa et al. 1986, Chaulet et al., 1990, Gava et al. 1991, 1996, Collazo \& RietCorrea 1996). Nesse trabalho não foi observada espongiose no sistema nervoso central, lesão secundária, principalmente, à hiperamonemia, causada pela distrofia hepática (síndrome hepato-cerebral). Essa ausência de lesões no sistema nervoso central pode ser explicada pela evolução clínica rápida e morte após curto período de sinais clínicos. Há também a possibilidade de que os sinais neurológicos possam estar relacionados à hipoglicemia (Norenberg \& Gregorius 1985), que é descrita em bovinos intoxicados por Xanthium spp. (Stuart et al. 1981, Witte et al. 1990, Martin et al. 1992). Neste estudo foi constatada acentuada hipoglicemia nos Bovinos 7 e 9. Sinais neuromusculares leves, no Bovino 7, foram observados com dosagem sérica de glicose dentro dos parâmetros normais. Porém, de forma semelhante ao Bovino 9, estavam coincidentemente acentuados com a constatação de hipoglicemia.
O principal achado de necropsia foi encontrado no fígado e consistia em aumento do padrão lobular na superfície capsular e de corte conferindo ao órgão aspecto de "nozmoscada". Neste trabalho experimental, o padrão lobular evidente está caracterizado por áreas alaranjadas claras intercaladas com áreas vermelho-escuras. Microscopicamente essa distribuição corresponde a áreas com hepatócitos morfologicamente normais ou com alterações degenerativas circundando áreas com hepatócitos apresentando alterações necróticas. Essa distribuição microscópica é característica de hepatotoxicoses agudas sendo descritas com frequiência em intoxicações por Xanthium spp. em bovinos (Martin et al. 1986, 1992, Witte et al. 1990, Méndez et al. 1998, Driemeier et al. 1999, Loretti et al. 1999), suínos (Stuart et al. 1981, Oelkers \& Oehme 1982) e ovinos (Loretti 1997). Esse padrão de lesão microscópica ocorre também em outras hepatopatias tóxicas em animais domésticos (Döbereiner et al. 1969, Tokarnia \& Döbereiner 1982, Jackson et al. 1984, Riet-Correa et al. 1986, Gava et al. 1987, 1991,1996, Dutra et al. 1997). Repleção da vesícula biliar, edemas teciduais, principalmente da parede da vesícula biliar e adjacências, e efusões cavitárias são observados com frequiência em animais domésticos intoxicados por Xanthium spp. (Stuart et al. 1981, Oelkers \& Oehme 1982, Martin et al. 1992, Loretti et al. 1999, Méndez et al. 1998, Driemeier et al. 1999).

Microscopicamente, o principal achado foi a necrose de coagulação zonal, centrolobular (Bov. 3 e 9) e panlobular (Bov. 7 e 10). A necrose hepática é causada por um princípio tóxico encontrado na brotação e frutos de Xanthium spp. Evidências de que o glicosídeo CAT fosse o princípio hepatotóxico foram obtidas com a reprodução experimental do quadro clínico-patológico em animais domésticos e de laboratório (Cole et al. 1980, Stuart et al. 1981, Seawright et al. 1982). O CAT inibe o mecanismo de fosforilação oxidativa, a síntese ou hidrólise de ATP e o transporte de ATP através da membrana mitocondrial induzindo à morte celular por anóxia (Seawright et al. 1982).

A biópsia do fígado, além de permitir a visualização microscópica de lesão hepática, permite avaliar a evolução das alterações morfológicas. Neste trabalho foram realizadas biópsias seqüienciais nos Bovinos 4, 6, 9 e 11. Nos Bovinos 6 e 11 não foram observadas alterações macro e microscópicas que pudessem ser atribuídas ao consumo de frutos de $X$. cavanillesii. No Bovino 9 as primeiras alterações microscópicas foram notadas 12 horas após a administração dos frutos, concomitante com a observação dos primeiros sinais clínicos, elevação dos níveis séricos de AST e hipoglicemia. As lesões microscópicas foram caracterizadas por leve aumento da acidofilia do citoplasma, picnose nuclear, dilatação dos sinusóides. Estas alterações são descritas na intoxicação por frutos de X. cavanillesii (Loretti 1997) e Microcystis aeruginosa (Jackson et al. 1984) em ovinos.

A realização de biópsia hepática neste experimento foi importante para estudar o desenvolvimento seqüencial das alterações morfológicas hepáticas relacionadas à intoxicação por $X$. cavanillesii em bovinos. Embora a técnica possa ser repetida muitas vezes em um mesmo animal sem que ocor- 
ram problemas, ela não é isenta de riscos. Complicações podem surgir com a perfuração de grandes vasos ou do ducto biliar da área portal, a perfuração de um abscesso hepático preexistente e em casos de coagulopatias, por lesão no parênquima hepático ou por alterações em outros sistemas, por exemplo, trombocitopenia (Kelly 1986). Por essa razão em tendências à hemorragia, recomenda-se verificar previamente a capacidade de coagulação. Porém se for considerado o curto período para o início dos sinais clínicos, a rápida evolução do quadro clínico, além dos custos econômicos, essa técnica não deve ser recomendada para a realização do diagnóstico de intoxicação por $X$. cavanillesii. Os dados epidemiológicos, sinais clínicos e estudo patológico dos animais mortos normalmente são suficientes para conclusão do diagnóstico. A realização de biópsia do fígado é melhor aplicável em doenças crônicas do fígado, nas quais o curso clínico é longo e a análise morfológica permite visualizar as lesões antes que os animais apresentem os sinais clínicos, diminuindo as perdas econômicas ou morte dos animais afetados (Driemeier 1990).

Agradecimentos.- Ao Professor Alexandre P. Loretti (UFRGS) pela colaboração e disponibilização de literatura sobre o assunto. Aos médicos veterinários Rosa Tasch (Cachoeira do Sul) e Flauri A. Migliavacca (Casca) pelas informações e colaboração na obtenção do resíduo de soja para o experimento.

\section{REFERÊNCIAS}

Braga M. B., Castilhos L.M.L. \& Santos M.N. 1985. Biópsia hepática em bovinos: proposta de nova técnica. Ciência Rural, Santa Maria, 15(1):79-88.

Canella C. F. C., Tokarnia C. H. \& Döbereiner J. 1968. Intoxicação por Sessea brasiliensis Toledo em bovinos. Pesq. Agropec. Bras. 3: 333-340.

Chaulet J. H. F., Peixoto, P. V. \& Tokarnia, C. H. 1990. Intoxicação experimental por Sessea brasiliensis (Solanaceae) em bovinos, ovinos e caprinos. Pesq. Vet. Bras. 10(3/4):71-74.

Cole R. J., Stuart B. P., Lansden, J. A. \& Cox, R. X. 1980. Isolation, and redefinition of the toxic agent from cocklebur Xanthium strumarium. J. Agric. Food Chem. 28:1330.

Collazo L. \& Riet-Correa F. 1996. Experimental intoxication of sheep and cattle with Wedelia glauca. Vet. Hum. Toxicol. 38(3):200-203.

Döbereiner J., Tokarnia C. H. \& Canella C. F. C. 1969. Intoxicação por Cestrum laevigatum Schlecht., a causa de mortandades em bovinos no Estado do Rio de Janeiro. Pesq. Agropec. Bras. 4:165-193.

Döbereiner J., Tokarnia C. H. \& Purisco E. 1976. Vernonia mollissima, planta tóxica responsável por mortandades de bovinos no sul do Mato Grosso. Pesq. Agropec. Bras. 11:49-58.

Driemeier D. 1990. Intoxicação experimental por Senecio oxyphyllus DC (Compositae) em bovinos. Tese de Mestrado, Universidade Federal de Santa Maria, Rio Grande do Sul. 107p.

Driemeier D., Irigoyen L. F., Loretti A. P. Colodel, E.M. \& Barros C. S. L. 1999. Intoxicação espontânea pelos frutos de Xanthium cavanillesii (Asteraceae) em bovinos no Rio Grande do Sul. Pesq. Vet. Bras. 19(1):12-18.

Dutra F., Riet-Correa F., Méndez M. C. \& Paiva N. 1997. Sawfly (Perreya flavipes) larvae poisoning of cattle and sheep in Uruguay. Vet. Hum. Toxicol. 39(5):281286.

Gava A., Peixoto P. V. \& Tokarnia C. H. 1987. Intoxicação experimental por Vernonia mollissima em ovinos e bovinos. Pesq. Vet. Bras. 7(2): 33-41.

Gava A., Stolf L., Pilati C., Neves D. S. \& Vigano L. 1991. Intoxicação por Cestrum corymbosum var. hirsutum (Solanaceae) em bovinos no Estado de Santa Catarina. Pesq. Vet. Bras. 11(3/4):71-74.

Gava A., Stolf L., Varaschin M. S., Neves D. S., Tigre A. P. \& Lessmann, F. 1996. Intoxicação por Cestrum intermedium (Solanaceae) em bovinos. Pesq. Vet. Bras. 16(4):117-120.

Jackson A. R. B., McInnes A., Falconer I. R. \& Runnegar M. T. C. 1984. Clinical and pathological changes in sheep experimentally poisoned by the bluegreen alga Microcystis aeruginosa. Vet. Pathol. 21:102-113.

Kelly W.R. 1986. Diagnóstico clínico veterinário. Panamericana, Rio de Janeiro. 364p.

Kingsbury J. M. 1964. Poisonous Plants of the United States and Canada. Prentice-Hall, New Jersey. 626p.

Loretti A. P. 1997. Intoxicação experimental pelos frutos de Xanthium cavanillesii (Asteraceae) em ovinos. Tese de Mestrado, Curso de Pós-Graduação em Medicina Veterinária, Universidade Federal de Santa Maria. 89p.

Loretti A.P., Bezerra P.S., Ilha M.R.S., Barros S.S. \& Barros C.S.L. 1999. Intoxicação experimental pelos frutos de Xanthium cavanillesii (Asteraceae) em ovinos. Pesq. Vet. Bras. 19(2):68-75.

Marsh C. D., Glenwood C. R. \& Clawson A. B. 1924. Cockleburs (species of Xanthium) as poisonous plants. Dept. Bull. 1274, U.S. Dept. Agriculture. 24p.

Martin T., Stair E. L. \& Dawson L. 1986. Cocklebur poisoning in cattle. J. Am. Vet. Med. Assoc. 189(5):562-563.

Martin T., Johnson B. J., Sangiah S. \& Burrows G. E. 1992. Experimental cocklebur intoxication (Xanthium strumarium) in calves, p. 489-494. In: James, L. F., Keeler, R. F., Bailey, E. M., Cheeke, P. R. Hegarty, M. P. (ed.) Poisonous Plants. Proc. 3rd Int. Symposium, Iowa University, Ames, lowa.

Méndez M. C., Santos R. C. \& Riet-Correa F. 1998. Intoxication by Xanthium cavanillesii in cattle and sheep in southern Brazil. Vet. Human Toxicol. 40(3):144-147.

Norenberg M.D. \& Gregorius J.B. 1985. Central nervous system manifestations of systemic disease, p. 403-467. In: Davis R.L. \& Robertson D.M. (ed.) Textbook of Neuropathology. Williams \& Wilkins, Baltimore.

Oelkers S. \& Oehme F. 1982. Cocklebur poisoning in swine. Bov. Pract. 3(2):1114.

Riet-Correa F, Schild A. L. \& Méndez M. C. 1986. Intoxicação por Cestrum parqui (Solanaceae) em bovinos no Rio Grande do Sul. Pesq. Vet. Bras. 6(4):111-115

Seawright A. A., Hrdlicka J., Lee J. A. \& Ogunsan E. A. 1982. Toxic substances in the food of animals: some recent findings of Australian poisonous plant investigation. J. Appl. Toxicol. 2:75.

Stuart B. P., Cole R. J. \& Gosser H. S. 1981. Cocklebur (Xanthium strumarium var. strumarium) intoxication in swine: review and redefinition of the toxic principle. Vet. Pathol. 18:368-383.

Tokarnia C. H. \& Döbereiner J. 1982. Intoxicação de bovinos por Vernonia rubricaulis (Compositae) em Mato Grosso. Pesq. Vet. Bras. 2(4):143-147.

Witte S. T., Osweiler G. D., Stahr H. M., Mobley G. 1990. Cocklebur toxicosis in cattle associated with the comsumption of Xanthium strumarium. J. Vet. Diagn. Invest. 2(4):263-267. 